\title{
Latest Results from MEG
}

\author{
Fabrizio $\mathrm{Cei}^{* \dagger}$ \\ INFN and University of Pisa, Dipartimento di Fisica, Largo Bruno Pontecorvo 3, 56127 Pisa, \\ Italy \\ E-mail: fabrizio.cei@pi.infn.it
}

We present the status and the perspectives of the MEG experiment which searched for the Lepton Flavour Violating decay $\mu^{+} \rightarrow \mathrm{e}^{+} \gamma$ for several years. In the full dataset corresponding to $7.4 \times$ $10^{14}$ positive muons stopped on target we didn't find any evidence for this decay and we set an upper bound on the $\mu^{+} \rightarrow \mathrm{e}^{+} \gamma$ branching ratio of $4.2 \times 10^{-13}$ at $90 \%$ C.L., with a sensitivity of $5.3 \times 10^{-13}$, which improves our previous world best limit by a factor of 1.5 and the best limit set by past experiments by a factor of 30. On the basis of the experience gained in MEG phase I we elaborated an upgrade plan of our detector, which was approved by the host laboratory and is presently in setting-up stage. We discuss here the status of the MEG upgrade (MEG II), which aims to improve the experimental sensitivity by a further order of magnitude.

XVII International Workshop on Neutrino Telescopes

13-17 March 2017

Venice, Italy

* Speaker.

${ }^{\dagger}$ On behalf of the MEG Collaboration. 


\section{Introduction to Lepton Flavour Violation and $\mu^{+} \rightarrow \mathrm{e}^{+} \gamma$ decay}

The Lepton Flavour Violation (LFV) in the charged lepton sector is almost forbidden in the Standard Model (SM), even including neutrino oscillations and mixing [1]. Conversely, the majority of New Physics models (NP) [2], particularly in view of recent measurements of a large $\theta_{13}$ at reactor [3] and accelerator [4] experiments, predict sizable branching ratios $(\mathscr{B})$ for LFV reactions as $\mu^{+} \rightarrow \mathrm{e}^{+} \gamma, \mu^{+} \rightarrow \mathrm{e}^{+} \mathrm{e}^{-} \mathrm{e}^{+}$and $\mu^{-} \rightarrow \mathrm{e}^{-}$conversion; for instance, the expected $\mathscr{B}\left(\mu^{+} \rightarrow \mathrm{e}^{+} \gamma\right)$ ranges from $\sim 10^{-14}$ to $\sim 10^{-12}$. The discovery of a LFV process would be a clear evidence for NP, whereas improvements in $\mathscr{B}$ Upper Limits (UL) would constitute significant constraints on the NP parameter space, complementary to those obtainable by direct searches at high energy colliders. Here we show the final results of the first phase and the perspectives of the upgrade of the MEG experiment, which improved the world UL on $\mathscr{B}\left(\mu^{+} \rightarrow \mathrm{e}^{+} \gamma\right)$ by a factor of 30 and can reach a sensitivity covering a large fraction of the parameter space allowed for NP schemes.

\section{The MEG experiment first phase: MEG I}

The MEG experiment [5] at Paul Scherrer Institute (PSI) [6] aims to search for the $\mu^{+} \rightarrow \mathrm{e}^{+} \gamma$ decay with a sensitivity $\sim 10^{-13}$ or better. The $\mu^{+} \rightarrow \mathrm{e}^{+} \gamma$ signature for muons decaying at rest is a back-to-back monoenergetic (52.83 MeV each) time-coincident $\mathrm{e}^{+}-\gamma$ pair. Positron and photon candidates are searched for by looking at their energies $\left(E_{\gamma}, E_{\mathrm{e}}\right)$, relative directions $\left(\theta_{\mathrm{e} \gamma}\right.$, $\left.\phi_{\mathrm{e} \gamma}\right)^{1}$ and emission time $\left(t_{\mathrm{e} \gamma}\right)$. The background has two components: the Radiative Muon Decay $\mu^{+} \rightarrow \mathrm{e}^{+} v_{\mathrm{e}} \bar{v}_{\mu} \gamma$ (RMD), whose rate, as that of the signal, is proportional to the $\mu$ stopping frequency $R_{\mu}$, and the ACCidental Background (ACCB), given by the random coincidence of an energetic $\mathrm{e}^{+}$from the SM decay $\mu^{+} \rightarrow \mathrm{e}^{+} v_{\mathrm{e}} \bar{v}_{\mu}$ (known as Michel decay) with a $\gamma$ from RMD, $\mathrm{e}^{+}-\mathrm{e}^{-}$annihilation in flight or bremsstrahlung. The ACCB, whose rate is proportional to $R_{\mu}{ }^{2}$, is responsible for $93 \%$ of MEG events with $E_{\gamma}>48 \mathrm{MeV}$. The RMD background in the MEG experiment was measured using data collected in 2009 and 2010, getting results in agreement with the SM predictions for $\mathrm{e}^{+}$and $\gamma$ energy spectra and angular distributions and for the absolute rate [7].

The first phase of the MEG experiment (MEG I) used the PSI $\pi$ E5 surface muon beam line, capable of delivering up to $10^{8}$ stopping $\mu^{+} / \mathrm{s}$ even if a lower intensity of $3 \times 10^{7} \mu^{+} / \mathrm{s}$ was used to take ACCB under control and avoid a worsening of the signal-to-noise ratio. Surface muons are produced with full polarization $\left(P_{\mu}=-1\right)$ and partially depolarized during the travel to the $\mu$ stopping target, where their residual polarization was measured to be $P_{\mu}=-0.86 \pm 0.05$, in agreement with expectations [8]. The $\mu^{+}$beam was stopped in a $205 \mu \mathrm{m}$ thick plastic target, slanted by $\approx 20^{\circ}$ with respect to the beam axis, and the $\gamma$ energy, arrival time and first interaction point and the $\mathrm{e}^{+}$track and timing were respectively measured by a Liquid Xenon (LXe) detector, seen by 846 UV-sensitive photomultipliers (PMTs) submerged in the liquid, and by a magnetic spectrometer composed by 16 Drift Chambers (DCH) and a double-array of scintillation Timing Counters (TC). The spectrometer was located inside a superconducting solenoid (COnstant Bending RAdius, COBRA) whose magnetic field is arranged to sweep out $\mathrm{e}^{+}$'s with small longitudinal momenta and to make the $\mathrm{e}^{+}$bending radius almost independent of the emission angle. The COBRA magnetic field

\footnotetext{
${ }^{1} \theta_{\mathrm{e} \gamma}=\left(\pi-\theta_{\mathrm{e}}\right)-\theta_{\gamma}$ and $\phi_{\mathrm{e} \gamma}=\left(\pi+\phi_{\mathrm{e}}\right)-\phi_{\gamma}, \theta$ and $\phi$ being the polar angle and the azimuthal angle respectively, taking the beam-axis as $z$-axis.
} 
was measured with a Hall probe on a grid of $1 \mathrm{~mm}$ spaced points and converted in a continuous 3-D function by a B-spline interpolation. The trigger system, based on FPGA technology, was designed to reduce the trigger rate from several $\mathrm{MHz}$ to $\sim 10 \mathrm{~Hz}$ by means of fast estimates of $\gamma$ energy, $\mathrm{e}^{+}-\gamma$ relative timing and direction, with a $>95 \%$ signal efficiency and a live time fraction of $\approx 99 \%$ [9]. The readout was performed by a custom-made chip (Domino Ring Sample, DRS), with a maximum sampling speed of $5 \mathrm{GHz}$ and 12 bit voltage digitization [10]. The detector was continuously monitored by a complex calibration system, extensively described in $[11,5,12]$.

\section{Data sample}

The experiment collected data continuously in stable running conditions since 2009 for five years. A first UL on $\mathscr{B}\left(\mu^{+} \rightarrow \mathrm{e}^{+} \gamma\right)$ was published in 2011 [13] based on the $2009-2010$ data sample and a much more significant one in 2013 [12], including also the data collected in 2011. Here we show the final results of MEG I, obtained by analyzing the full dataset, corresponding to $\approx 7.4 \times 10^{14}$ positive muons stopped on target, a sample twice larger than that used for 2013 result.

\section{Analysis procedure and results}

The MEG analysis, described in detail in [13], is based on a combination of a blind and a maximum likelihood (ML) approach, applied in the analysis region defined by $48 \mathrm{MeV}<E_{\gamma}<$ $58 \mathrm{MeV}, 50 \mathrm{MeV}<E_{\mathrm{e}}<56 \mathrm{MeV},\left|t_{\mathrm{e} \gamma}\right|<0.7 \mathrm{~ns},\left|\theta_{\mathrm{e} \gamma}\right|<50 \mathrm{mrad}$ and $\left|\phi_{\mathrm{e} \gamma}\right|<50 \mathrm{mrad}$. We call "time sidebands" the regions defined by $1 \mathrm{~ns}<\left|t_{\mathrm{e} \gamma}\right|<4 \mathrm{~ns}$, " $E_{\gamma}$-sideband" that defined by $40 \mathrm{MeV}<E_{\gamma}<48 \mathrm{MeV}$ and "angle sidebands" those defined by $50 \mathrm{mrad}<\left|\phi_{\mathrm{e} \gamma}\right|<150 \mathrm{mrad}$ or $50 \mathrm{mrad}<\left|\theta_{\mathrm{e} \gamma}\right|<150 \mathrm{mrad}$. Positron and photon reconstruction are discussed in detail in [12].

The $\mathrm{e}^{+}$track is reconstructed by using a Kalman filter track fitting technique [14], supplemented by the GEANE package [15] for hit modelling, multiple scattering and energy loss. The track is propagated to the TC and the track fitting is iteratively refined by including the timing information. As a by-product, a parameter covariance matrix and a per-track error are extracted, which are included in the MEG likelihood function. Tracks with $\geq 7$ hits and $\leq 2$ turns in the spectrometer are retained for the analysis and a single $\mathrm{e}^{+}$per event is selected by applying other track quality cuts. The track is then back extrapolated to the target to determine the $\mathrm{e}^{+}$vertex. The resolutions on $\mathrm{e}^{+}$energy, $\mathrm{e}^{+}$polar angles and vertex coordinates [13] are $\sigma_{E_{\mathrm{e}}} \approx(310-330) \mathrm{keV}$ at $E_{\mathrm{e}}=52.83 \mathrm{MeV}, \sigma_{\phi_{\mathrm{e}}} \approx(7-8) \mathrm{mrad}^{2}$ at $\phi_{\mathrm{e}}=0, \sigma_{\theta_{\mathrm{e}}} \approx(10-11) \mathrm{mrad}, \sigma_{z} \approx(1.5-2.0) \mathrm{mm}$ in the beam direction and $\sigma_{y} \approx(1.2-1.3) \mathrm{mm}$ in the vertical direction. In the LXe $\gamma$ detector the $\gamma$ timing, first interaction point and total energy release are measured by looking at the pattern of scintillation light detected by the PMTs near the incident position, at the leading edge times of the PMT waveforms and at the total collected charge. Special algorithms are used to identify pile-up events $(\approx 15 \%$ of the total) and single out the individual contributions of all $\gamma$ 's forming the pile-up combination; the charge integration window can then be re-adjusted, improving the energy resolution. The $\gamma$ reconstruction efficiency, estimated by MC simulation and measured with $55 \mathrm{MeV}$ $\gamma$ 's from $\pi^{0}$ decay, was $\sim 63 \%$. The $\gamma$ position, timing and energy resolutions were repeatedly

\footnotetext{
${ }^{2}$ The ranges here and later on take into account the yearly differences caused by various factors (e.g. variable DCH configurations and efficiencies).
} 
measured by using the charge exchange reaction $\pi^{-} \mathrm{p} \rightarrow \pi^{0} \mathrm{n}$, followed by the $\pi^{0}$ decay in two $\gamma^{\prime} \mathrm{s}$, whose energy can be selected to be $\approx 55 \mathrm{MeV}$ or $\approx 83 \mathrm{MeV}$ by placing an auxiliary detector (a $\mathrm{NaI}$ or a BGO array) in back-to-back coincidence with the LXe calorimeter. Such resolutions are respectively: $\sigma_{y} \approx 5 \mathrm{~mm}$ on the $\gamma$ entrance face and $\sigma_{r} \approx 6 \mathrm{~mm}$ along the radial depth, $\sigma_{t} \approx 67 \mathrm{ps}$ on $\gamma$ timing and $\sigma_{E} / E \approx(1.7-2.4) \%$ (depending on the event depth) on relative energy resolution. The energy scale stability was also continuously monitored [5] and re-adjusted to maintain the overall spread within $1 \%$. The resolutions on the relative directions were determined by combining the corresponding $\mathrm{e}^{+}$and $\gamma$ resolutions obtaining $\approx 16 \mathrm{mrad}$ for $\theta_{\mathrm{e} \gamma}$ and $\approx 9 \mathrm{mrad}$ for $\phi_{\mathrm{e} \gamma}$. The relative time $t_{\mathrm{e} \gamma}$ was measured from the RMD peak observed in the $E_{\gamma}$-sideband above the flat ACCB distribution obtaining $\sigma_{t_{\mathrm{er}}}=130 \pm 2 \mathrm{ps}$, with the most important contributions coming from the uncertainties on $\mathrm{e}^{+}$track length $(\sim 75 \mathrm{ps})$ and on TC $(\sim 65 \mathrm{ps})$ and $\mathrm{LXe}(\sim 67 \mathrm{ps})$ intrinsic time resolutions. The position of the RMD-peak $\left(t_{\mathrm{e} \gamma}=0\right)$ was stable within 15 ps during the whole data taking period. The relative alignment between the DCHs was obtained by looking at straight tracks from cosmic muons and at curved $\mathrm{e}^{+}$tracks and comparing the measurements with an optical survey $[5,12]$. The relative alignment between the LXe detector and the spectrometer was determined by looking at AIF events (see later) and at cosmic ray tracks without the magnetic field, with a precision of $\sim 0.5 \mathrm{~mm}$ in the longitudinal ( $z$ ) direction. Finally, the position of the target relative to the DCH system was determined by using optical alignment techniques and software tools. Several cross marks on the target foil were monitored year by year with a theodolite, providing estimated accuracies of $0.5 \mathrm{~mm}$ in direction orthogonal to the beam and of $1.5 \mathrm{~mm}$ along the beam direction. The target position was checked on a large sample of back propagated $\mathrm{e}^{+}$tracks comparing the reconstructed positions of a group of holes on the target surface with the nominal ones.

Our analysis algorithms were significantly improved [16] after the publication of 2013 results [12]. First of all, we developed a technique to identify tracks which cross the target twice, with the first turn not correctly reconstructed (Missing First Turn, (MFT) events). When the first turn is missed, the $\mathrm{e}^{+}$track length and time of flight are underestimated, producing a some percent inefficiency in the selection of $\mu^{+} \rightarrow \mathrm{e}^{+} \gamma$ candidates. Fig. 1 (left) shows an example of how MFT recovery algorithm works: a $\mathrm{e}^{+}$was originally reconstructed as a double-turn track, but the MFT recovery algorithm identified a missing first turn and the $\mathrm{e}^{+}$track was refitted and classified as a triple-turn one. The $\mu^{+} \rightarrow \mathrm{e}^{+} \gamma$ selection efficiency improved by $\approx 4 \%$. Secondly, we developed a new algorithm to single out events where a $\mathrm{e}^{+}$annihilates in flight on an $\mathrm{e}^{-}$(AIF events). Such events can be identified by the presence of a track which stops in the DCH system, in angular correlation with a $\gamma$ line-of-flight drawn by the track end point (the AIF vertex) to the impact point of a $\gamma$ in the LXe detector, as shown in Figure 1 (right). The identification of AIF events produced a $1.1 \%$ signal inefficiency and a $1.9 \%$ background reduction, an effect not so marginal as it appears, since the rare AIF events produce the largest contribution to the upper side of the $\gamma$ energy spectrum and a single non identified AIF event can significantly worsen the UL on $\mathscr{B}\left(\mu^{+} \rightarrow \mathrm{e}^{+} \gamma\right)$. Third (last, but not least) we measured a year-dependent shift (up to a couple of $\mathrm{mm}$ ) of the target position along the beam axis and a continuous deformation of the target itself, possibly due to radiation damage and plastic material deterioration. Because of this deformation the target planarity was lost and the target surface was approximated by a paraboloidal surface and sampled with a few $\mu \mathrm{m}$ grid by a 3-D FARO laser scanner [17]. The true and approximate target shapes are shown in Fig. 2. These analysis refinements motivated the collaboration to reprocess the full dataset from scratch 

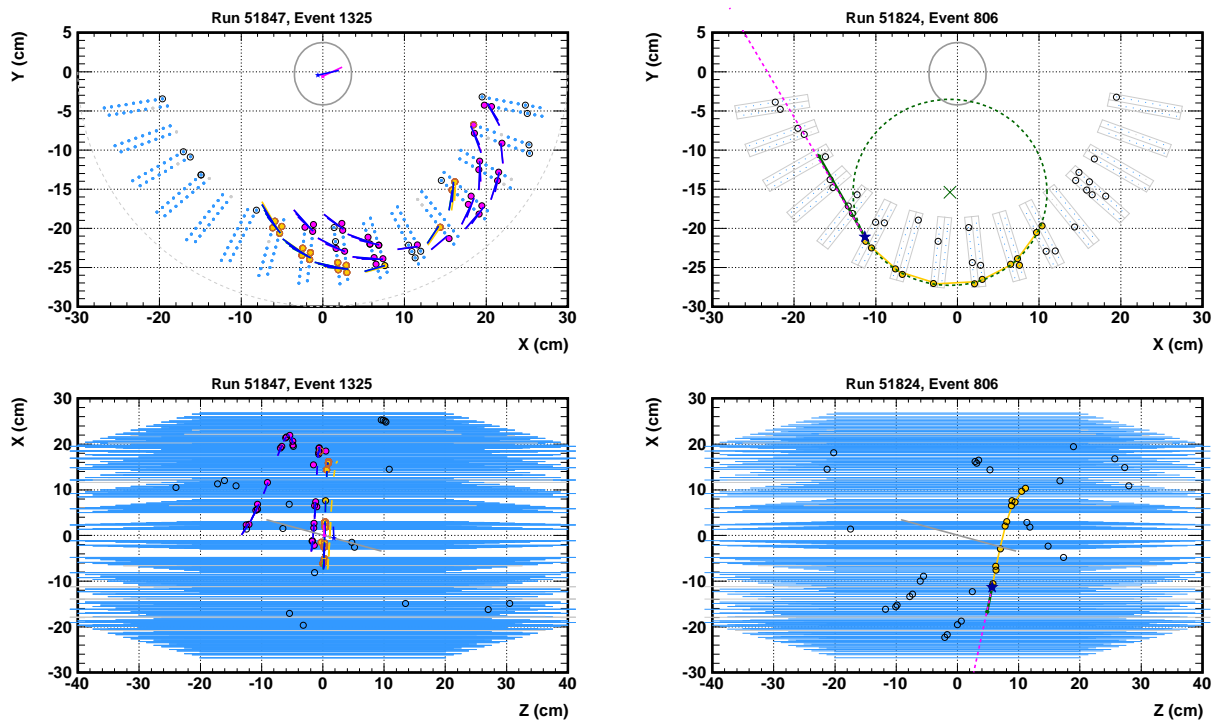

Figure 1: Left: the MFT recovery algorithm at work. The $\mathrm{e}^{+}$track, originally reconstructed as a doubleturn one (magenta hits), is refitted and classified as a triple-turn track by the MFT recovery algorithm, which identifies a track segment (the brown hits) as the missing first turn of this $\mathrm{e}^{+}$. Right: example of an AIF candidate event. The AIF vertex is indicated by a blue star, visible in the upper plot at $(x, y)=(-12,-21)$. The AIF direction is indicated by a green arrow and the $\gamma$ line-of-flight by a magenta line. Note that green arrow and magenta line nearly overlap, confirming the interpretation of this event as due to AIF.
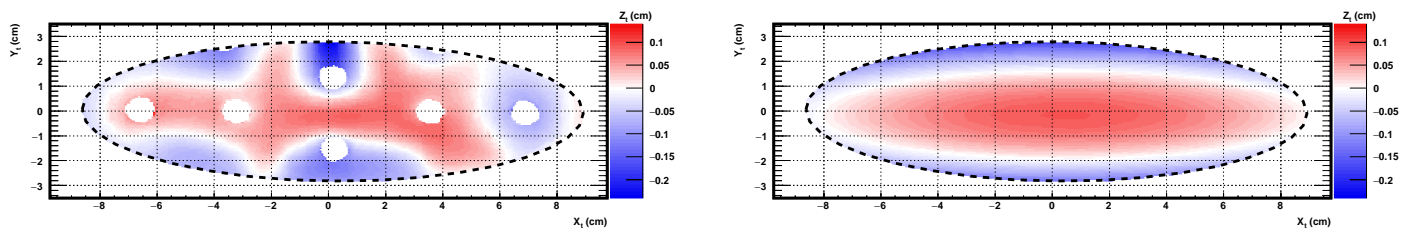

Figure 2: The FARO scan measurement (left) of the target shape and the paraboloid fit for 2013 (right). The maximum discrepancies between the measurements and the approximate shape are $\sim \pm 0.2 \mathrm{~mm}$, but most of the events are concentrated in the central region of the target, where the difference is much smaller.

and to check the consistency with previously published results obtained on reduced samples.

The ML analysis is supplemented by a blind analysis procedure, where events with $48 \mathrm{MeV}<$ $E_{\gamma}<58 \mathrm{MeV}$ and $\left|t_{\mathrm{e} \gamma}\right|<1 \mathrm{~ns}$ are hidden until the Probability Density Functions (PDFs) for the likelihood function are finalized. Time, energy and angle sidebands are used to optimize the analysis, study ACCB and extract the corresponding PDFs, while Signal (S) and RMD events PDFs are determined by using calibration data and measured resolutions. Two different types of PDFs are extracted, one based on per-event errors, called per-event PDFs, and one based on event cathegories (high and low quality tracking, deep or shallow energy deposition within the LXe detector ...), called constant PDFs. Per-event PDFs use relative polar angles $\theta_{\mathrm{e} \gamma}$ and $\phi_{\mathrm{e} \gamma}$ independently, while constant PDFs use the stereo angle difference $\Theta_{\mathrm{e} \gamma}$. Two analysis groups processed the data, one with one set of PDFs and one with the other, and compared their results. The per-event analysis, which exploited the entire information, had a slightly better sensitivity and is considered as the official MEG analysis. All the PDFs were inserted in the global likelihood function [16] which took 
into account the poissonian fluctuation of the total number of events and the sideband constraints on the number of RMD and ACCB events and was modified to include the year dependent target displacement and deformation in form of a nuisance parameter vector. The number of S, RMD and ACCB events in the analysis region and the year-by-year nuisance parameters were extracted by a ML fit. The confidence interval and the UL on the number of $S$ events were calculated by using the Feldman-Cousins frequentist method [13, 18], with the numbers of RMD and ACCB events left free to fluctuate. The experimental sensitivity $\mathscr{S}_{90}$ was preliminarly evaluated by generating a large sample of pseudo experiments (toy MCs) with 0 signal events and fitting them with the ML algorithm. $\mathscr{S}_{90}$ is defined as the median of the distribution of the $90 \%$ C.L. ULs extracted from the toy MC ensemble. Events falling in the timing sidebands, where no signal events can be present, were also fit with the same technique, getting ULs in good agreement with $\mathscr{S}_{90}$. The normalization factor to convert an UL on the number of S events into an UL on $\mathscr{B}\left(\mu^{+} \rightarrow \mathrm{e}^{+} \gamma\right)$ was computed by counting the number of Michel $\mathrm{e}^{+}$'s selected with a dedicated pre-scaled trigger or the number of RMD events observed in the muon data and combining the uncertainties. The two methods exhibited a pretty good agreement, as shown in Figure 3 (left). The combined uncertainty on the
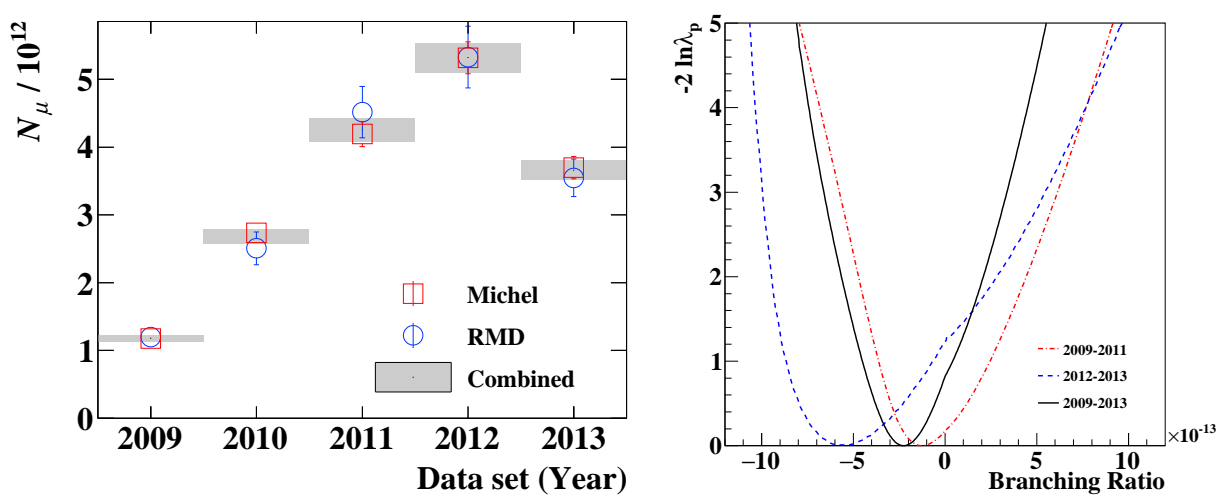

Figure 3: Left: the number of stopped muons on target calculated by counting the number of Michel or RMD events and their weighted average year by year. Right: the negative log-likelihood ratio $\lambda_{p}$ as a function of the signal branching ratio.

$\mathscr{B}$ value was $3.5 \%$. Systematic uncertainties on PDF parameters and on normalization were taken into account in the calculation of the confidence intervals by fluctuating the PDFs by the amount of the uncertainties. Fig. 4 shows the event distributions in the $\left(E_{\mathrm{e}}, E_{\gamma}\right)$ and $\left(\cos \Theta_{\mathrm{e} \gamma}, t_{\mathrm{e} \gamma}\right)$ planes for the MEG full dataset, together with the contours of the averaged signal PDFs at 1, 1.64 and $2 \sigma$. We show in Fig. 5 the results of the ML fit for the full dataset 2009-2013: the best fit for $\mathscr{B}\left(\mu^{+} \rightarrow \mathrm{e}^{+} \gamma\right)$ was $-2.2 \times 10^{-13}$, showing no evidence for $\mu^{+} \rightarrow \mathrm{e}^{+} \gamma$ events. The plots on the top line and on the left and center bottom line show the distributions of the five observables $t_{\mathrm{e} \gamma}, E_{\mathrm{e}}, E_{\gamma}$, $\theta_{\mathrm{e} \gamma}$ and $\phi_{\mathrm{e} \gamma}$, while the plot on the bottom right shows the distribution of $R_{\text {sig }}$ variable, defined as the logarithmic ratio of the $\mathrm{S}$ likelihood and of the background likelihood, a weighted average of RMD and ACCB likelihoods. We performed also separate ML fits for the previously published sample (2009 - 2011) and for the data never analysed before (2012 - 2013), getting for the former sample a result statistically consistent with the previous one. The observed profile likelihood ratios as a function of $\mathscr{B}$ for the full 2009 - 2013 dataset, the $2009-2011$ sample used in [12] analysis and 

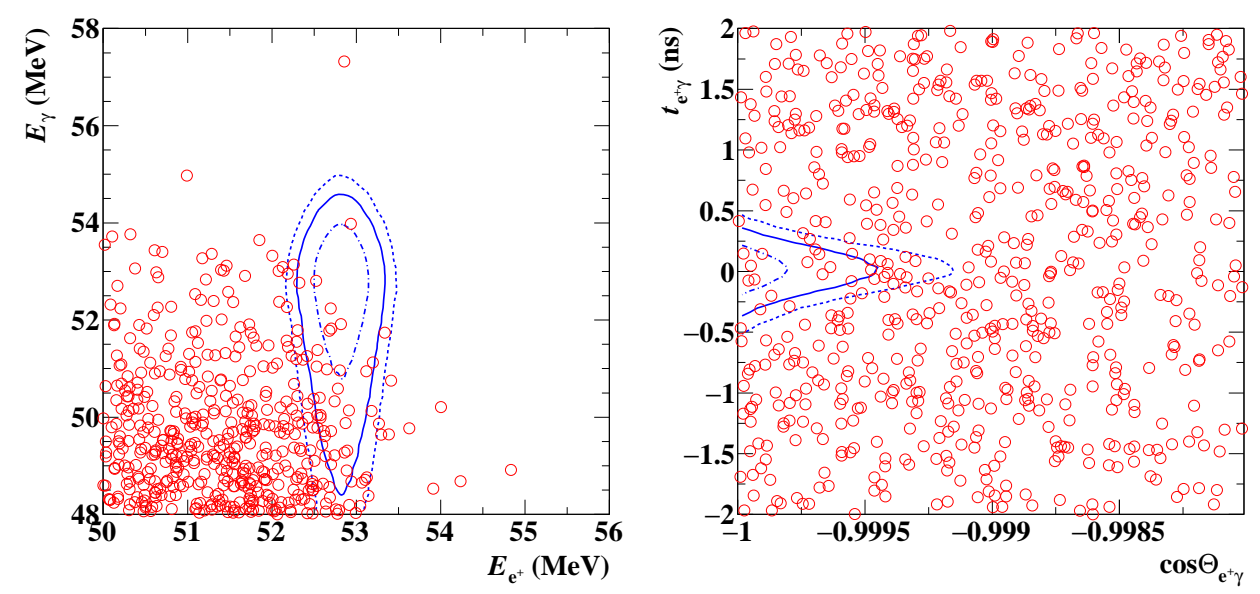

Figure 4: Event distributions for the full dataset in the $\left(E_{\mathrm{e}}, E_{\gamma}\right)$ and $\left(\cos \Theta_{\mathrm{e} \gamma}, t_{\mathrm{e} \gamma}\right)$ planes. In the figure on the left (right) a selection of $\left|t_{\mathrm{e} \gamma}\right|<0.244 \mathrm{~ns}$ and $\cos \Theta_{\mathrm{e} \gamma}<-0.9996$ with $90 \%$ efficiency for each variable $\left(52.4 \mathrm{MeV}<E_{\mathrm{e}}<55 \mathrm{MeV}\right.$ and $51 \mathrm{MeV}<E_{\gamma}<55.5 \mathrm{MeV}$ with $90 \%$ and $74 \%$ efficiencies for $E_{\mathrm{e}}$ and $E_{\gamma}$, respectively) is applied. The signal PDF contours $(1,1.64$ and $2 \sigma)$ are also shown.

Table 1: Best fit value ( $\mathscr{B}_{\text {fit }}$ 's), branching ratio Upper Limit $\left(\mathscr{B}_{90}\right)$ and sensitivity $\left(\mathscr{S}_{90}\right)$ for three datasets: full $(2009-2013)$ and partial $((2009-2011)$ and $(2012-2013))$ data samples.

\begin{tabular}{cccc}
\hline Dataset & $\mathscr{B}_{\text {fit }} \times 10^{13}$ & $\mathscr{B}_{90} \times 10^{13}$ & $\mathscr{S}_{90} \times 10^{13}$ \\
\hline $2009-2011$ & -1.3 & 6.1 & 8.0 \\
$2012-2013$ & -5.5 & 7.9 & 8.2 \\
$2009-2013$ & -2.2 & 4.2 & 5.3 \\
\hline
\end{tabular}

the $2012-2013$ (new data) dataset are shown in Fig. 3 (right). The best $\mathscr{B}$ fit ( $\mathscr{B}$ fit), UL at $90 \%$ C.L. $\left(\mathscr{B}_{90}\right)$ and $\mathscr{S}_{90}$ for these samples are listed in Table $1 . \mathscr{B}_{90}$ and $\mathscr{S}_{90}$ for the full $2009-2013$ dataset are respectively $4.2 \times 10^{-13}$ and $5.3 \times 10^{-13}$. An independent ML fit based on constant PDFs gave pretty consistent results: $\mathscr{B}_{\text {fit }}$ and $\mathscr{B}_{90}$ for the $\mu^{+} \rightarrow \mathrm{e}^{+} \gamma$ decay were $-2.5 \times 10^{-13}$ and $4.3 \times 10^{-13}$ in very good agreement with the per-event analysis. We show in Fig. 6 (left) the distribution of the relative stereo angle $\Theta_{\mathrm{e} \gamma}$ used in the constant PDF analysis instead of $\theta_{\mathrm{e} \gamma}$ and $\phi_{\mathrm{e} \gamma}$ separately. The consistency between the two analyses was checked on a large sample of toy MCs, as shown in Fig. 6 (right). The final MEG result, published in 2016 [16], improved the previous MEG UL [12] by a factor of 1.5 and the UL on $\mathscr{B}\left(\mu^{+} \rightarrow \mathrm{e}^{+} \gamma\right)$ set by past experiments by a factor $\approx 30[19]$.

\section{The MEG upgrade: MEG II}

The final result of MEG I represents a strong constraint on all NP models; however, it should be advisable to push down the UL on $\mathscr{B}\left(\mu^{+} \rightarrow \mathrm{e}^{+} \gamma\right)$ by a further order of magnitude if this can be obtained with moderate costs and on a short time-scale $(\sim 3-4$ years). This requires a significant upgrade of MEG detector (called MEG II), with substantial re-designs of some parts of the experiment, in order to overcome the limitations observed in MEG I and improve the performances of various sub-detectors. This should result in a better background reduction and could allow to 

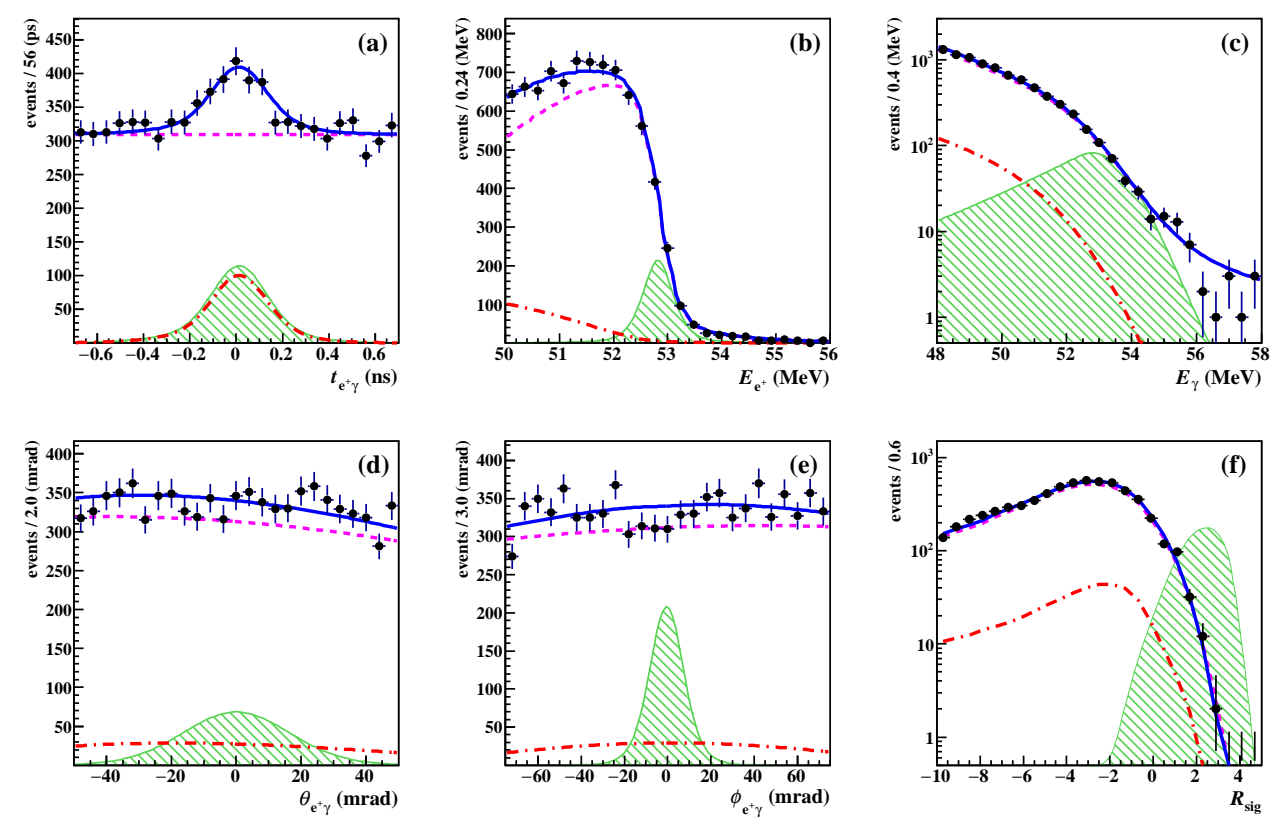

Figure 5: Results of ML fit for the 2009-2013 dataset for the five observables: $t_{\mathrm{e} \gamma}, E_{\mathrm{e}}, E_{\gamma}, \theta_{\mathrm{e} \gamma}$ and $\phi_{\mathrm{e} \gamma}$. The black dots are the data and the colour lines represent the contributions extracted from the fit: RMD (red), ACCB (purple) and total (blue), while the green hatched histogram represents the signal UL at $90 \%$ C.L. magnified by 100 . The plot on the right bottom shows the distribution of the relative signal likelihood $R_{s i g}$.

take advantage of a higher intensity muon beam (from $3 \times 10^{7}$ to $7 \times 10^{7}$ stopping $\mu^{+} / \mathrm{s}$ ) and of an enlarged detector acceptance. A schematic view of the MEG II detector is shown in Fig. 7 (left).

The most important and most challenging part of the upgrade is the replacement of the MEG I DCH system with a highly transparent (the amount of material traversed by a $52.83 \mathrm{MeV} \mathrm{e}^{+}$corresponds to $\left.<2 \times 10^{-3} X_{0}\right), 2 \mathrm{~m}$ long unique volume cylindrical drift chamber $(\mathrm{CDCH})$, with a $2 \pi$ azimuthal coverage and equipped with $\approx 1300$ sense wires and $\sim 8000$ field and guard wires. The $\mathrm{CDCH}$ has a $(7-8)^{\circ}$ stereo view angle and will be operated with a $85: 15 \mathrm{He} / \mathrm{C}_{4} \mathrm{H}_{10}$ gas mixture. The advantages of this new detector with respect to the MEG I segmented DCH system are higher momentum and angular resolution and an about doubled $\mathrm{e}^{+}$efficiency: such better performances come from the elimination of dead zones where the $\mathrm{e}^{+}$'s can stop (for instance the plastic cathodic frames of the DCH system), from the much larger number of measurement points along the track ( $\sim 60$ instead of $\sim 10$ ), from the much better coupling with the TC, located just below the chamber frame, and from the elimination of the cathode foils for the $z$ coordinate reconstruction, which caused problems of low level signals and electric discharges. The expected single hit resolution on the radial view $(120 \mu \mathrm{m})$ and on the $z$ coordinate $(\sim 10 \mathrm{~cm}$, measured by timing differences and charge division on the wire ends) were verified with laboratory measurements on several prototypes by using cosmic rays and beam tests [21]. The ageing of the gas mixture was also measured, with the encouraging conclusions that the expected gain drop during the MEG II DAQ time $(\sim 40 \%)$ can be corrected by a moderate increase of the HV. The chamber is presently in assembling phase in the INFN Pisa and Lecce laboratories under controlled environmental conditions.

The other major upgrades of MEG II involve: a) a thinner target (140 $\mu \mathrm{m}$ thickness), placed 

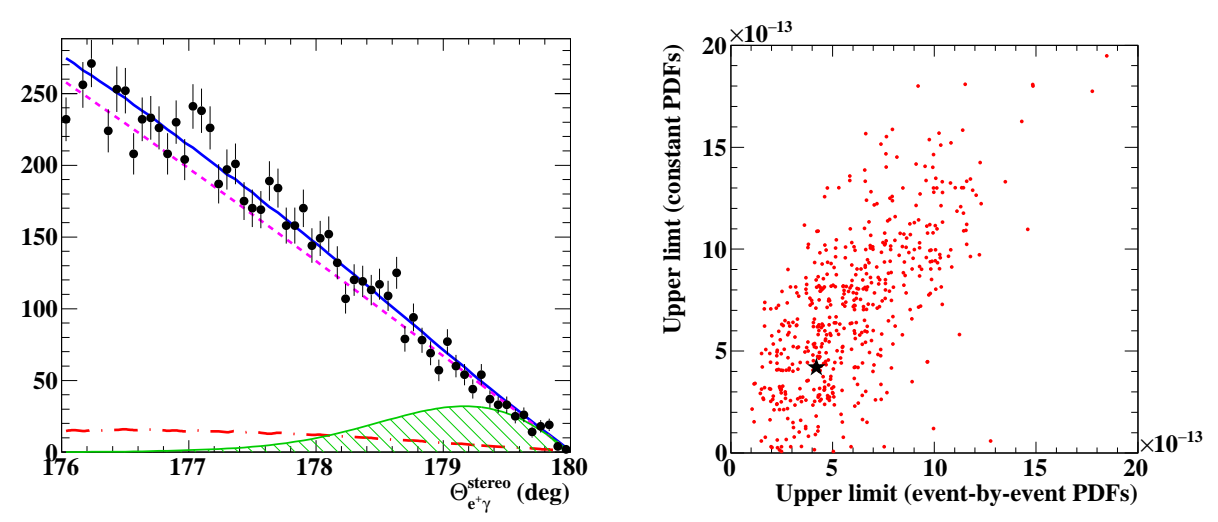

Figure 6: Left: distribution of $\Theta_{\mathrm{e} \gamma}$ variable as extracted by the constant PDF analysis. Right: comparison between the $90 \%$ C.L. ULs obtained by fitting a big sample of toy MC experiments by the per-event analysis (horizontal axis) and the constant PDF analysis (vertical axis). The two analyses are clearly correlated, with a $20 \%$ better sensitivity for the more refined per-event analysis. The black star represents the real experiment.
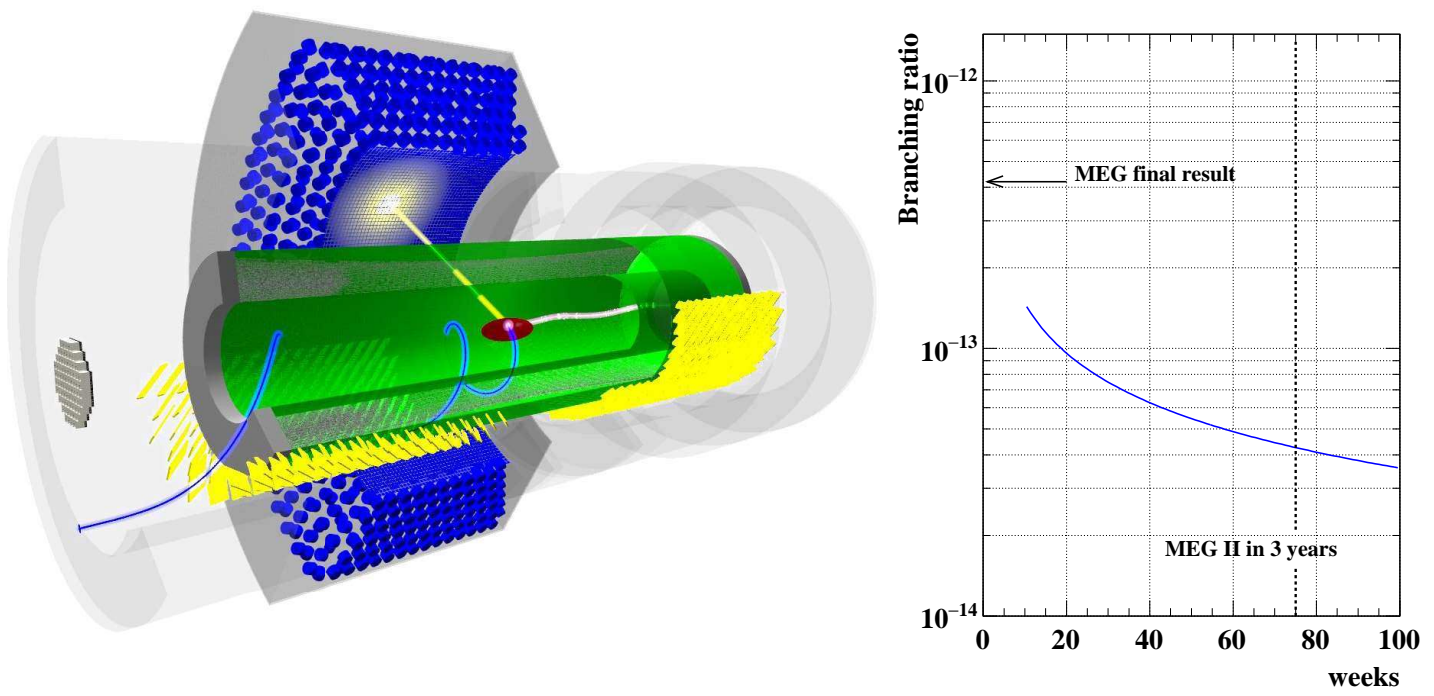

Figure 7: Left: schematic view of the MEG II detector. Right: expected sensitivity of MEG upgrade as a function of DAQ time. The MEG I final upper limit [16] is indicated by a black arrow. Assuming 25 weeks of data taking per year, we expect to reach a sensitivity on $\mu^{+} \rightarrow \mathrm{e}^{+} \gamma$ branching ratio of $\approx 4.3 \times 10^{-14}$.

at a $15^{\circ}$ slant angle, to better substain the increased $\mu$ stopping rate; b) the replacement of the LXe $\gamma$ detector inner face PMTs with an array of $409212 \mathrm{~mm} \times 12 \mathrm{~mm}$ UV-sensitive Silicon Photomultipliers (SiPM) to increase the granularity and the fraction of active surface coverage and enhance the light collection efficiency, the pile-up rejection capability and the uniformity of the detector response. A factor 2 improvement in energy and position resolution is expected, particularly important for events where the $\gamma$ converts very close to the entrance window. The $\gamma$ detection efficiency is also expected to increase by $\sim 5 \%$ because of the smaller mass to be traversed by $\gamma$ 's entering the LXe detector. All SiPM are installed and under testing with LED light and ra- 
dioactive sources; c) a redesign of the LXe detector lateral sides and a better placement of the PMTs mounted on them to enlarge the acceptance and make the light collection more uniform. The PMT mounting is complete; d) the building of a new pixelated TC, formed by 2-D arrays of scintillator tiles, to improve the $\mathrm{e}^{+}$timing measurement resolution by means of multiple hits in different tiles. A complete TC module, assembled in November 2016, was tested in engineering runs, where the expected average number of crossed tiles $(8-9)$ and the corresponding timing resolution $(\approx 35 \mathrm{ps})$ were experimentally confirmed; e) the building of a new multi-functional DAQ board (WaveDREAM) which integrates analog frontend, trigger, digitization and HV, designed to fulfill the requests of an increased number of read-out channels and of a higher bandwidth; $f$ ) the insertion in the downstream side of the experiment of a LYSO+plastic scintillator detector to detect low energy $\mathrm{e}^{+}$'s emitted in RMD and tag the high-energy $\gamma$ coming from the same decay (RDC counter). This detector is ready and was succesfully tested at PSI; a $40-48 \%$ identification efficiency is expected. We show in Fig. 8 a pictorial view of the MEG II detector elements under construction: most of them are close to completion and the final assembling is envisaged within 2017. The figures of merit of the upgraded detector are compared in Table 2 with that of MEG I.

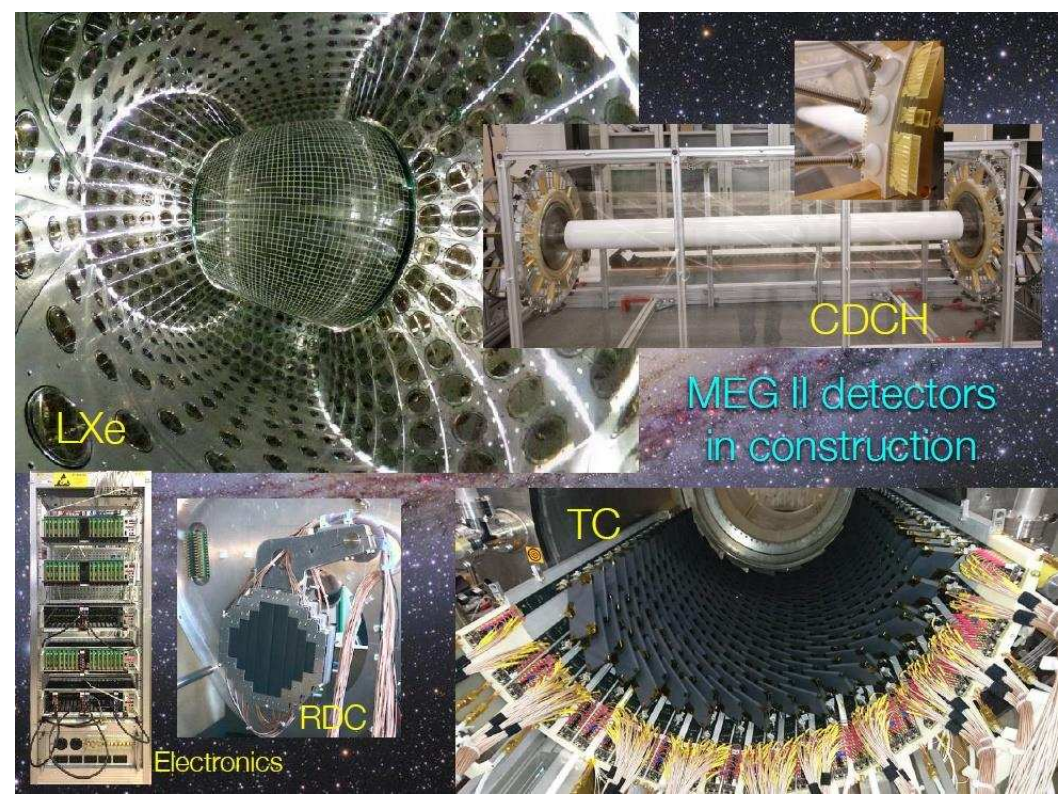

Figure 8: Pictorial view of MEG II sub-detectors under construction.

The MEG II expected sensitivity as a function of the DAQ time is shown in Fig. 7 (right). In 3 years of data taking we expect to reach a sensitivity of $\approx 4.3 \times 10^{-14}$ with an improvement of one order of magnitude with respect to the final sensitivity reached by MEG I.

\section{References}

[1] F. Böhm \& P. Vogel, Physics of Massive Neutrinos, Cambridge University Press, (1992), S. T. Petcov, Sov. J. Nucl. Phys. 25 (1977) 340.

[2] R. Barbieri, L. Hall and A. Strumia, Nucl. Phys. B 455 (1995) 219, J. Hisano, D. Nomura and T. Yanagida, Phys. Lett. B 437 (1998) 351, M. Raidal et al., Eur. Phys. J. C 57 (2008) 13,

G. Blankenburg et al., Eur. Phys. J. C 72 (2012) 2126. 
Table 2: Resolutions (Gaussian $\sigma$ ) and efficiencies for MEG II compared with MEG I

\begin{tabular}{lll}
\hline PDF parameters & MEG I & Upgrade MEG II \\
\hline$\sigma_{E_{\mathrm{e}^{+}}}(\mathrm{keV})$ & 380 & 130 \\
$\mathrm{e}^{+} \sigma_{\theta} / \sigma_{\phi}(\mathrm{mrad})$ & $9.4 / 8.7$ & $5.3 / 3.7$ \\
$\mathrm{e}^{+} \sigma_{Z} / \sigma_{Y}(\mathrm{core})(\mathrm{mm})$ & $2.4 / 1.2$ & $1.6 / 0.7$ \\
$\frac{\sigma_{E_{\gamma}}}{E_{\gamma}}(\%) w>2 \mathrm{~cm}$ & 1.7 & 1.0 \\
$\gamma$ position at $\mathrm{LXe} \sigma_{(u, v)} / \sigma_{w}(\mathrm{~mm})$ & $5 / 6$ & $2.6 / 5$ \\
$\gamma-\mathrm{e}^{+}$timing $(\mathrm{ps})$ & 122 & 84 \\
\hline Efficiency $(\%)$ & & \\
\hline trigger & $\approx 99$ & $\approx 99$ \\
$\gamma / \mathrm{e}^{+}$reconstruction & $63 / 30$ & $69 / 70$ \\
event selection & 80 & 85 \\
\hline
\end{tabular}

[3] F. P. An et al., Phys. Rev. Lett. 108 (2012) 171803, F. P. An et al., Phys. Rev. Lett. 112 (2014) 061801, J. K. Ahn et al., Phys. Rev. Lett. 108 (2012) 191802, Y. Abe et al., Phys. Lett. B 723 (2013) 66.

[4] K. Abe et al., Phys. Rev. Lett. 107 (2011) 041801, K. Abe et al., Phys. Rev. Lett. 112 (2014) 061802.

[5] J. Adam et al., Eur. Phys. J. C 73 (2013) 2365

[6] http://www.psi.ch

[7] A. Baldini et al., Eur. Phys. J. C 76 (2016) 108

[8] A. Baldini et al., Eur. Phys. J. C 76 (2016) 223

[9] L. Galli et al., JINST 8 (2013) P01008, L. Galli et al., JINST 9 (2014) P04022.

[10] S. Ritt et al., Nucl. Instr. Meth. A 623 (2010) 486

[11] J. Adam et al., Nucl. Instr. Meth. A 641 (2011) 19, A. M. Baldini et al., Nucl. Instr. Meth. A 565 (2006) 589, A. M. Baldini et al., Nucl. Instr. Meth. A 545 (2006) 753, G. Rutar et al., Nucl. Instr. Meth. A 824 (2016) 575

[12] J. Adam et al., Phys. Rev. Lett. 110 (2013) 201801

[13] J. Adam et al., Phys. Rev. Lett. 107 (2011) 171801

[14] P. Billoir, Nucl. Instr. Meth. A 225 (1984) 352, R. Frühwirth, Nucl. Instr. Meth. A 262 (1987) 444.

[15] V. Innocente and E. Nagy, Nucl. Instr. Meth. A 324 (1993) 297, A. Fontana et al., J. Phys. Conf. Ser. 119 (2008) 032018.

[16] A. Baldini et al., Eur. Phys. J. C 76 (2016) 434

[17] FARO portable coordinate measuring machines http;//www.faro.com/en-us/products

[18] J. Beringer et al. (Particle Data Group), Phys. Rev. D 86 (2012) 010001, G. J. Feldman and R.D. Cousins, Phys. Rev. D 57 (1998) 3873.

[19] M. Ahmed et al., Phys. Rev. D. 65 (2002) 112002.

[20] A. M. Baldini et al., MEG Upgrade Proposal, physics.ins-det/1301 . 7225.

[21] M. Venturini et al., Nucl. Instr. Meth. A 824 (2016) 589-591. 